\title{
Valoración multidimensional del envejecimiento en la ciudad de Antofagasta
}

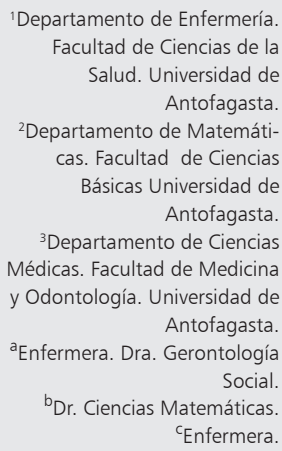

Financiamiento: Proyecto DI 1327. Financiado por concurso interno de la Dirección Genera de Investigación. Universidad de Antofagasta.

Recibido el 16 de abril de 2009, aceptado el 15 de enero de 2010.

Correspondencia a: Catalina Tapia Pinto Avenida Angamos № 601 Antofagasta. Chile. Teléfono: 55- 637422/55 637230 - Fax 55637802 E-mail: ctapia@uantof.cl

\author{
CATALINA TAPIA P. ${ }^{\text {a }}$, HÉCTOR VARELA V ${ }^{2 b}$ \\ LUIS BARRA A. ${ }^{3}$, M. DOLORES UBILLA V..$^{\text {lc }}$, VERÓNICA ITURRA M. ${ }^{1 c}$, \\ CARMEN COLLAO A. ${ }^{\text {lc }}$, ROSA SILVA Z. ${ }^{\text {lc }}$
}

\section{Cross sectional geriatric assessment of free living older subjects from Antofagasta, Chile}

Background: The effects of aging on people must be evaluated to adequate sanitary actions. Aim: To assess the characteristics of older subjects living in Antofagasta, Chile. Material and Methods: Cross sectional assessment of 602 subjects without dementia, aged more than 60 years (55\% females). A socioeconomic and medical history was obtained and Barthel, Lawton, Yesavage depression, Tromp fall risk and Folstein Mini mental scales were applied. Results: Sixty eight percent of subjects had more than six years of studies. Forty six percent had hypertension, $28 \%$ had osteoarticular problems, $20 \%$ had hypercholesterolemia and $17 \%$ diabetes mellitus. Four percent had depression and falls were reported by 35\%. Seventy four percent were independent in basic and instrumental activities. Ninety four percent had normal cognitive functions. Age was an important determinant of functional capacity. Conclusions: The main problems detected in this sample were the risk of falls and the presence of chronic diseases.

(Rev Med Chile 2010; 138: 444-451).

Key words: Geriatrics; Accidental fall; Aging.
L a distribución geográfica del proceso de transición al envejecimiento demográfico no es homogénea en el país ${ }^{1}$. Así, el índice nacional de adultos mayores (AM) para 2010 será de 60 AM por cada 100 menores de 15 años; Antofagasta sólo presentará un índice de 40. La diferencia en este índice se mantiene y, según proyecciones para el año $2020^{2}$, mientras Antofagasta registrará 58 AM, a nivel nacional será de 85 y Valparaíso llegará a 103 AM. Los datos demuestran lo heterogéneo que se distribuye geográficamente el envejecimiento en el país. Otra particularidad es la esperanza de vida a los 60 años, que entre 2005 y 2010, a nivel nacional, es 19,5 para los hombres y 22,4 para las mujeres; mientras la región de Antofagasta ${ }^{3}$ presenta 17,6 y 21,4 respectivamente, valor más bajo en el país. La región de Antofagasta, tanto en el índice de AM, como en la esperanza de vida a los 60 años, presenta cifras menores a nivel nacional. Ambos datos deben responder a múltiples factores, aún desconocidos.

El propósito básico es investigar cómo se encuentran los AM de una ciudad que a nivel regional presenta las características señaladas en el párrafo anterior, avalado en la necesidad de reconocer diferencias contextuales del proceso de envejecimiento. Es importante considerar las relaciones que existen entre ambiente y vejez. Se plantea que la forma de envejecer depende, entre otros aspectos, del lugar en que se vive y la estimulación fisicosocial que recibe el $\mathrm{AM}^{4}$. Además, el impacto del contexto social influye en las cohortes, las que son afectadas por eventos y cambios sociales ocurridos en un momento histórico. 
Otro propósito del estudio es una característica de la gerontología, el intervencionismo, cuyo propósito es mejorar las condiciones de vida que permitan a los AM un envejecimiento satisfactorio. Previo a cualquier intervención, se debe identificar el fenómeno de envejecimiento para posteriormente intervenir sobre los aspectos que resulten en déficit ${ }^{5}$.

Desde Tartler en 1961 (teoría de la actividad), hasta la recomendación actual de la Organización Mundial de la Salud ${ }^{6}$ sobre el envejecimiento activo, definido como: "el proceso de optimización de las oportunidades en relación con la salud, la participación y la seguridad para mejorar la calidad de vida a medida que se envejece", se reconoce el impacto de la salud, para que el AM se mantenga con independencia y autonomía a nivel personal, familiar y sociocomunitario. Este estudio incorpora aspectos cualitativos como autopercepción de salud, que expresa la sensación de bienestar individual y es aceptado como buen indicador del estado global de ésta; además de la autopercepción de memoria. Finalmente, por constituirse en un factor que puede modificar tanto a nivel cualitativo como objetivo el estado de salud ${ }^{7}$, se consultó la presencia de enfermedades crónicas que afectan la calidad de vida del AM.

Esta investigación se realiza en dos fases, estos datos corresponden a la primera, en la cual se efectuó una valoración geriátrica multidimensional.

\section{Material y Método}

El diseño fue descriptivo correlacional. Los objetivos son: 1) describir características sociales, biológicas y psicológicas de los AM de la ciudad de Antofagasta y 2) correlacionar las características biosicosociales con el rango de edad, indicador de la cohorte generacional. El universo en estudio para la ciudad fue 20.290 AM. Se trabajó con una muestra aleatoria estratificada con afijación proporcional. Los estratos fueron dos por género y tres por rango de edad. El tamaño de los estratos, se obtuvo de la información del censo 2002 (INE). El tamaño de muestra calculado con $95 \%$ de confianza y un margen de error en la estimación de 0,04 corresponde a 602 personas de 60 y más años. El instrumento recolector de datos fue una encuesta que correspondía a una valoración geriátrica. Se usó el índice de Barthel para actividades básicas de la vida diaria, en la versión de $\mathrm{Shah}^{8}$ (1989); el rango global de puntuaciones varía entre 0 (dependiente total) y 100 (independiente). Se utilizó la escala de Lawton-Brody ${ }^{8}$ para las actividades instrumentales, cuyo puntaje varía entre 1 (dependencia total), 2 (dependencia parcial) y 3 (independencia). La depresión se evaluó con la escala de depresión geriátrica de Yesavage ${ }^{8}$; en su versión abreviada (GDS); el puntaje mínimo es 0 (sin depresión) y 15 el máximo (depresión establecida). Para la evaluación de deterioro cognitivo se utilizó el Minimental State Examination de Folstein ${ }^{8}$ (MMS); puntaje mínimo 0 y máximo 30. El punto de corte para letrados se fijo en 24 y en 20 para analfabetos ${ }^{8}$. El riesgo de caídas múltiples se evaluó a través de la escala de Tromp y col. Esta incluye la presencia de caída en los últimos 12 meses, con el máximo puntaje (5); los problemas visuales (4); la limitación funcional e incontinencia se puntúan 3 puntos cada una. El puntaje varía entre 0 y 15 puntos; el corte está establecido en 7 , a partir del cual se considera que el riesgo de caídas es alto9 . La encuesta incluía datos sociodemográficos y del estado de salud como son: presencia de enfermedades crónicas (se solicitaba algún documento que confirme la información) y consumo fármacos. La autopercepción del estado de salud y memoria se midió a través de escala tipo Likert. La fiabilidad (Cronbach) de las escalas es: Barthel (,812); Lawton-Brody (,829); Yesavage (,839); Minimental State Examination de Folstein (,730); riesgo de caídas de Tromp (,672). La recolección de datos la realizaron internos de $5^{\circ}$ año de la carrera de enfermería, previa preparación y acreditación de trabajo con AM autónomos. Los datos se obtuvieron en el sector norte, centro y sur de Antofagasta, desde consultorios de atención primaria, grupos comunitarios deportivos, religiosos, folklóricos, sociales y culturales. El criterio de exclusión fue evidencia de deterioro cognitivo; evaluado con el Short Portable Mental State Questionnaire de Pfeiffer por rápido y sencillo para un screening. El AM que contestaba las cinco primeras preguntas erróneamente, se clasificaba con deterioro y no se aplicaba el instrumento ${ }^{8}$. La cantidad de AM excluidos fue escaso, pero no se tiene una cifra exacta. Este estudio fue evaluado y aprobado por el Comité de Ética de la Universidad de Antofagasta.

Estadística: Primero se realizó análisis descriptivo. Posteriormente, al comparar los puntajes me- 
dios de las escalas de evaluación geriátrica según los grupos etáreos por el método de análisis de varianza (ANOVA), se encontró que no se satisfacían los supuestos habituales para la validez de las conclusiones a través de este análisis. Se realizó la prueba de Kruskal-Wallis para comparar puntajes medianos y realizar un análisis de relación entre el rango de edad y la puntuación total de las escalas de evaluación geriátrica. Se utilizó SPSS para Windows, versión 11.5.

\section{Resultados}

Los principales rasgos descriptivos fueron: género femenino alcanzó $54,5 \%$. El rango de edad de 60-69 años obtuvo 56,1\%. Casados, 49,2\% y viudos, $32,4 \%$. El 72,6\% vivía acompañado; 93\% tenía hijos; $87,7 \%$ nietos y $39,0 \%$, bisnietos. El nivel educacional con mayor representación fue primario y secundario completo (Tabla 1). Al recategorizar nivel de escolaridad en menos y más de 6 años, esta última categoría alcanzó 67,8\%. Las patologías crónicas con mayor frecuencia fueron: 46,3\% hipertensión arterial (HTA); 27,9\% problemas osteoarticulares; $19,9 \%$ hipercolesterolemia y diabetes mellitus $17,7 \%$. El 33,9\% presentaba HTA y diabetes mellitus, patologías que asociadas presentan mayor impacto en la salud. También se observó alto consumo de fármacos diarios (Tabla 2). Las auto-percepciones (Tabla 3 ) en relación al estado de salud y memoria en la categoría "Buena percepción" fueron: $44,2 \%$ y 49,2\% respectivamente. El 56,7\% no percibía limitaciones para las actividades de la vida diaria.

El 74,1\%, para la escalas de Barthel y Lawton, determinaron mayor distribución porcentual para la categoría independiente (Tabla 4). La función cognitiva, evaluada por el MMS, determinó que $94 \%$ no presentaba deterioro, sin embargo, se excluyó AM que presentaban evidencia de deterioro (Tabla 5). La aplicación de la GDS, que orienta la sospecha de depresión, se observan en la Tabla 6. Las caídas estaban presentes en 34,6 (Tabla 7).

Se compararon los puntajes medianos de las escalas de evaluación geriátrica, según los rangos de edad. Dado que la diferencia observada entre las tres medias de rangos fue estadísticamente significativa, se puede plantear que la distribución de los puntajes en las evaluaciones geriátricas es diferente según los rangos de edad (Tabla 8). Este análisis se realizó también con la cohorte generacional con resultados similares, lo que evidencia la estrecha relación entre ambos indicadores. El estudio de la capacidad cognitiva debe considerar la influencia de la escolaridad. Así, se analizó la relación entre menos y más de 6 años de escolaridad, y la MMS

Tabla 1. Distribución porcentual de características biodemográficas de adultos mayores de la ciudad de Antofagasta. 2007

\begin{tabular}{|c|c|c|c|}
\hline Variables & & $\mathbf{n}$ & $\%$ \\
\hline \multirow[t]{2}{*}{ Sexo } & Hombre & 274 & 45,5 \\
\hline & Mujer & 328 & 54,5 \\
\hline Rango & $60-69$ & 338 & 56,1 \\
\hline \multirow[t]{2}{*}{ edad } & $70-79$ & 198 & 32,9 \\
\hline & 80 y más & 66 & 11,0 \\
\hline \multirow[t]{4}{*}{ Estado civil } & Soltero & 51 & 8,5 \\
\hline & Casado & 296 & 49,2 \\
\hline & Viudo & 195 & 32,4 \\
\hline & Separado & 60 & 10,0 \\
\hline \multirow{10}{*}{$\begin{array}{l}\text { Nivel } \\
\text { educacional }\end{array}$} & Sin estudio & 4 & 0,7 \\
\hline & $\begin{array}{l}\text { Sin estudios pero lee } \\
\text { y escribe }\end{array}$ & 18 & 3,0 \\
\hline & $\begin{array}{l}\text { Estudios primarios } \\
\text { incompletos }\end{array}$ & 86 & 14,3 \\
\hline & $\begin{array}{l}\text { Estudios primarios } \\
\text { completos }\end{array}$ & 121 & 20,1 \\
\hline & $\begin{array}{l}\text { Estudios secundarios } \\
\text { incompletos }\end{array}$ & 103 & 17,1 \\
\hline & $\begin{array}{l}\text { Estudios secundarios } \\
\text { completos }\end{array}$ & 121 & 20,1 \\
\hline & $\begin{array}{l}\text { Estudios técnicos } \\
\text { incompletos }\end{array}$ & 23 & 3,8 \\
\hline & $\begin{array}{l}\text { Estudios técnicos } \\
\text { completos }\end{array}$ & 70 & 11,6 \\
\hline & $\begin{array}{l}\text { Estudios universitarios } \\
\text { incompletos }\end{array}$ & 8 & 1,3 \\
\hline & $\begin{array}{l}\text { Estudios universitarios } \\
\text { completos }\end{array}$ & 48 & 8,0 \\
\hline \multirow{8}{*}{$\begin{array}{l}\text { Estructura } \\
\text { social }\end{array}$} & Vive acompañado & 437 & 72,6 \\
\hline & Vive solo & 165 & 27,4 \\
\hline & Tiene hijos & 560 & 93,0 \\
\hline & No tiene hijos & 42 & 7,0 \\
\hline & Tiene nietos & 528 & 87,7 \\
\hline & No tiene nietos & 74 & 22,3 \\
\hline & Tiene bisnietos & 235 & 55,6 \\
\hline & No tiene bisnietos & 335 & 39,0 \\
\hline
\end{tabular}


Valoración multidimensional del envejecimiento en la ciudad de Antofagasta - C. Tapia P. et al

Tabla 2. Distribución porcentual de características de salud en $\mathbf{6 0 2}$ adultos mayores de la ciudad de Antofagasta. 2007

\begin{tabular}{|lcc|}
\hline Patologías & n & $\%$ \\
\hline Hipertensión arterial & 279 & 46,3 \\
\hline $\begin{array}{l}\text { Problemas osteoarticualres (artrosis, } \\
\text { artritis, osteoporosis y deformidades }\end{array}$ & 168 & 27,9 \\
articulares) & & \\
Hipercolesterolemia & 120 & 19,9 \\
\hline Diabetes & 105 & 17,4 \\
Digestivas (estreñimiento, úlcera & 83 & 13,8 \\
gástrica y duodenal) & & \\
Cardiovasculares (insuficiencia & 66 & 11,0 \\
cardíaca, angina, bloqueos, arritmias) & & \\
Visión & 192 & 31,9 \\
Hipoacusia & 46 & 7,6 \\
Patologías asociadas & & \\
Sin HTA y diabetes & 398 & 66,1 \\
Con HTA y diabetes & 204 & 33,9 \\
Total & 602 & 100 \\
\hline Consumo diario de fármacos & 105 & 17,4 \\
No consume & 149 & 24,8 \\
Consume un fármaco/día & 133 & 22,1 \\
Consume dos fármacos/día & 108 & 17,9 \\
Consume tres fármacos/día & 107 & 17,8 \\
Consume 4 - 7 fármacos/día & 100 \\
\hline Total & & \\
\hline
\end{tabular}

Tabla 3. Distribución porcentual de autopercepciones del estado de salud en adultos mayores de la ciudad de Antofagasta. 2007

\begin{tabular}{|llrc|}
\hline & & n & \% \\
Autopercepción & Mala & 36 & 6,0 \\
del estado de & Muy mala & 14 & 2,3 \\
salud & Regular & 220 & 36,5 \\
& Buena & 266 & 44,2 \\
& Muy buena & 66 & 11,0 \\
& Total & 602 & 100 \\
Autopercepción & Mala & 34 & 5,6 \\
de memoria & Muy mala & 6 & 1,0 \\
& Regular & 190 & 31,6 \\
& Buena & 296 & 49,2 \\
& Muy buena & 76 & 12,6 \\
Autopercepción & Total & 602 & 100 \\
\cline { 2 - 2 } & Poca limitación & 341 & 56,7 \\
actividades vida & Regular limitación & 106 & 17,7 \\
diaria & Mucha limitación & 54 & 16,6 \\
& Total & 601 & 100 \\
\hline
\end{tabular}

Tabla 4. Valoración geriátrica funcional de los adultos mayores de la ciudad de Antofagasta. Escala de Barthel (modificación Shah) y Lawton Brody. 2007

\begin{tabular}{|c|c|c|c|c|c|c|c|c|}
\hline \multirow[t]{2}{*}{ Escalas } & \multicolumn{2}{|c|}{ Independencia } & \multicolumn{2}{|c|}{$\begin{array}{c}\text { Dependencia } \\
\text { escasa-moderada }\end{array}$} & \multicolumn{2}{|c|}{$\begin{array}{l}\text { Dependencia } \\
\text { severa-total }\end{array}$} & \multicolumn{2}{|c|}{ Total } \\
\hline & $\mathbf{n}$ & $\%$ & $\mathbf{n}$ & $\%$ & n & $\%$ & n & $\%$ \\
\hline Barthel & 446 & 74,1 & 149 & 24,7 & 7 & 1,2 & 602 & 100 \\
\hline Lawton-Brody & 446 & 74,1 & 140 & 23,2 & 16 & 2,7 & 602 & 100 \\
\hline
\end{tabular}

Tabla 5. Valoración cognitiva de los adultos mayores de la ciudad de Antofagasta. Minimental State Examination de Folstein (MMS) 2007

\begin{tabular}{|lcc|}
\hline Escala MMS & n & \% \\
\hline Con deterioro cognitivo & 36 & 6,0 \\
\hline Sin deterioro cognitivo & 566 & 94,0 \\
\hline Total & 602 & 100 \\
\hline
\end{tabular}

Tabla 6. Valoración de depresión geriátrica en los adultos mayores de la ciudad de Antofagasta. Escala depresión geriátrica (Yesavage). 2007

\begin{tabular}{|lrc|}
\hline Escala GDS & $\mathbf{n}$ & \% \\
\hline Sin depresión & 515 & 86 \\
\hline Depresión leve & 61 & 10 \\
\hline Depresión establecida & 26 & 4 \\
\hline Total & 602 & 100 \\
\hline
\end{tabular}


Tabla 7. Valoración del riesgo de caídas múltiples en los adultos mayores de la ciudad de Antofagasta. Escala de Tromp y col. 2007

\begin{tabular}{|lrc|}
\hline $\begin{array}{l}\text { Escala riesgos de caídas } \\
\text { múltiples }\end{array}$ & $\mathbf{n}$ & \% \\
\hline Sin riesgos & 279 & 46,3 \\
\hline Riesgo regular & 115 & 19,1 \\
\hline Riesgo alto & 76 & 12,6 \\
\hline Riesgo muy alto & 132 & 22,0 \\
\hline Total & 602 & 100 \\
\hline
\end{tabular}

categorizado en Sin y Con deterioro, que determinó una relación con significancia estadística, con un $\mathrm{p}=, 000$ (Tabla 9). Considerando que bajos niveles de escolaridad pueden resultar falsos diagnósticos de deterioro, se estudió la relación entre escolaridad y la escala de Lawton-Brody; los resultados evidenciaron mayor distribución porcentual para la dependencia funcional en menos de 6 años de escolaridad, con una significancia estadística avalada con un $\mathrm{p}=, 001$, (Tabla 10). Por tanto, se observó mayor frecuencia de deterioro cognitivo y funcional en personas con menos educación.

Tabla 8. Prueba de Kruskal-Wallis. Escalas de evaluación geriátrica y rango de edad, en adultos mayores de la ciudad de Antofagasta. 2007

\begin{tabular}{|lccc|}
\hline & Rangos de edad & n & Rango promedio \\
\hline Puntaje total & $60-69$ & 338 & 266,40 \\
Tromp & $70-79$ & 198 & 341,47 \\
& $80-$ más & 66 & 361,35 \\
Puntaje total & Total & 602 & \\
Barthel & $60-69$ & 338 & 329,30 \\
& $70-79$ & 198 & 280,96 \\
& $80-$ más & 66 & 220,77 \\
\hline Puntaje total & Total & 602 & \\
Lawton & $60-69$ & 338 & 335,11 \\
& $70-79$ & 198 & 291,12 \\
& $80-$ más & 66 & 160,52 \\
\hline Puntaje total & Total & 602 & 272,23 \\
GDS & $60-69$ & 338 & 336,15 \\
& $70-79$ & 197 & 343,41 \\
Puntaje total & $80-$ más & 66 & 290,44 \\
MMS & Total & 601 & 185,43 \\
& $60-69$ & 338 & \\
\hline & $70-79$ & 198 & 330,64 \\
& $80-$ más & 602 & \\
\hline
\end{tabular}

Estadístico de contraste

\begin{tabular}{|lccccc|}
\hline & $\begin{array}{c}\text { Total } \\
\text { MMS }\end{array}$ & $\begin{array}{c}\text { Total } \\
\text { Lawton }\end{array}$ & $\begin{array}{c}\text { Total } \\
\text { Barthel }\end{array}$ & $\begin{array}{c}\text { Total } \\
\text { GDS }\end{array}$ & $\begin{array}{c}\text { Total } \\
\text { Tromp }\end{array}$ \\
\hline Chi cuadrado & 25,905 & 95,577 & 43,299 & 22,667 & 35,832 \\
Gl & 2 & 2 & 2 & 2 & 2 \\
\hline Sig. asintót. & .000 & .000 & .000 & .001 & .000 \\
\hline
\end{tabular}

a) Prueba de Kruskal-Wallis. b) Variable de agrupación: rango edad. 
Valoración multidimensional del envejecimiento en la ciudad de Antofagasta - C. Tapia P. et al

Tabla 9. Minimental State Examination (Folstein) según escolaridad en adultos mayores de la ciudad de Antofagasta

\begin{tabular}{|lcccccc|}
\hline Escolaridad & \multicolumn{2}{c}{ Minimental } & \multicolumn{2}{c|}{ Examination } & \multicolumn{3}{c|}{ State } \\
(años) & \multicolumn{2}{c}{ Con deterioro } & \multicolumn{2}{c}{ Sin deterioro } & \multicolumn{2}{c|}{ Total } \\
& $\mathbf{n}$ & $\%$ & $\mathbf{n}$ & $\mathbf{\%}$ & $\mathbf{n}$ & $\%$ \\
Menos de 6 años & 22 & 61,1 & 172 & 30,4 & 194 & 32,2 \\
Más de 6 años & 14 & 38,9 & 394 & 69,6 & 408 & 67,8 \\
\hline Total & 36 & 100 & 566 & 100 & 602 & 100 \\
\hline
\end{tabular}

Chi cuadrado de 14.627. $p=, 000$.

Tabla 10. Actividades instrumentales (Lawton-Brody) según escolaridad en adultos mayores de la ciudad de Antofagasta

\begin{tabular}{|c|c|c|c|c|c|c|c|c|}
\hline \multirow{3}{*}{ Escolaridad } & \multicolumn{6}{|c|}{ Actividades instrumentales (Lawton-Brody) } & \multicolumn{2}{|c|}{ Total } \\
\hline & \multicolumn{2}{|c|}{ Independencia } & \multicolumn{2}{|c|}{$\begin{array}{c}\text { Dependencia } \\
\text { escasa-moderada }\end{array}$} & \multicolumn{2}{|c|}{$\begin{array}{l}\text { Dependencia } \\
\text { severa total }\end{array}$} & \multirow[b]{2}{*}{$\mathbf{n}$} & \multirow[b]{2}{*}{$\%$} \\
\hline & $\mathbf{n}$ & $\%$ & $\mathbf{n}$ & $\%$ & $\mathbf{n}$ & $\%$ & & \\
\hline Menos de 6 años & 128 & 28,7 & 56 & 40 & 10 & 62,5 & 194 & 32,2 \\
\hline Más de 6 años & 318 & 71,3 & 84 & 60 & 6 & 37,5 & 408 & 67,8 \\
\hline Total & 446 & 100 & 140 & 100 & 16 & 100 & 602 & 100 \\
\hline
\end{tabular}

Chi cuadrado de 13.128. $p=, 001$.

\section{Discusión}

La muestra proviene de una población con el menor índice de AM a nivel nacional, así, 56,1\% presenta entre 60 y 69 años. En esta región, hasta los 59 años, el sexo masculino es porcentualmente mayor, pero esta situación se invierte a los 60 años, lo que implica mayor presencia femenina.

El promedio de los años de estudios a nivel nacional, para los 60-64 años, es de 8,36 y 8,26 para los 65-69 años; la región de Antofagasta presenta 9,02 y 8,47 , respectivamente, superando la media nacional. Entre los 70 y 79 años, la región presenta una media menor, no obstante, a los 80 y más años se supera la media nacional de 9,69 a 10,67. Estos datos reflejan el 44,8\% con estudios secundarios hasta universitarios. Esta situación se presenta en los grandes centros urbanos como Antofagasta ${ }^{10}$.

El alto porcentaje que vive acompañado, así como la existencia de hijos, nietos y bisnietos, demuestra cambios descritos teóricamente ${ }^{11}$ en la estructura familiar, asociado al envejecimiento.
El principal problema de salud corresponde a HTA, presente en $46,3 \%$, valor que para los $60 \mathrm{y}$ más años es más bajo en comparación con la Encuesta Nacional de Salud (2003), que entregó una prevalencia de 53,7\% (45-64 años) y de 78,8\% (65 y más años $)^{12}$. El 33,9\% presenta diabetes mellitus e HTA en conjunto. Es decir, la situación de salud es similar a otras regiones con mayor índice de $\mathrm{AM}^{13-14}$. Esto puede indicar envejecimiento con mayor riesgo de fragilidad por comorbilidad en Antofagasta ${ }^{15}$, además, implica un mayor riesgo frente a diversos problemas de salud.

Las caídas alcanzan $34,6 \%$, el proyecto internacional "SABE" encontró una prevalencia de caídas para Chile de 34\%; el valor encontrado en esta investigación es levemente superior ${ }^{16}$ podría sugerir la presencia de factores intrínseco (patologías, consumo de fármacos, haber sufrido caídas) y extrínseco, como barreras arquitectónicas ${ }^{17}$.

La alta capacidad funcional $(74,1 \%)$ observada en las actividades básicas de la vida diaria e instrumentales, corresponde a: los participantes 
son activos, no presentan limitaciones severas de movilidad, participan en la comunidad, por lo tanto, conservan la capacidad para su cuidado cotidiano $^{18}$. El 56,1\% tiene entre 60 y 69 años y la dependencia para actividades del diario vivir se presenta alrededor de los 75-80 años ${ }^{19}$. El 2,7\% de dependencia en las actividades instrumentales indica pérdida en el manejo independiente de los AM, que incrementará al aumentar la edad.

Al comparar los puntajes medianos de las diferentes escalas, se observó que el rango de edad marca diferencias significativas en las capacidades funcionales y cognitivas, así como la presencia de depresión y riesgo de caídas de los AM. En las escalas de Lawton y Barthel, los rangos promedios disminuyen al aumentar la edad, lo que implica pérdida de funcionalidad. Se observó una mayor diferencia significativa entre los rangos 60-69 y 80 y más años en ambas escalas, lo que confirmaría que a mayor edad, aumenta la dificultad para el desempeño de las actividades instrumentales y básicas, generando dependencia. Esta relación entre mayor edad y limitación en las actividades básicas e instrumentales, se reportó en todas las ciudades participantes del proyecto $\mathrm{SABE}^{16}$. Esta pérdida de funcionalidad implicaría pérdida de la calidad de vida en los AM autónomos. El riesgo de caídas, presentó un rango promedio mayor en el rango 80 y más años, que para los otros rangos de edad; es decir, aumenta la edad y el riesgo. El deterioro cognitivo fue más bajo que el publicado en otros estudios, se debe tener presente el alto nivel de escolaridad, factor determinante para disminuir el riesgo de deterioro cognitivo. Concordante con otros estudios ${ }^{20}$, se encontró que menos de 6 años de escolaridad se asoció con deterioro en alto porcentaje $(61,1 \%)$ y con dependencia funcional instrumental $(62,5 \%)$. La pérdida de capacidad funcional es uno de los criterios del DSM-IV ${ }^{21}$ para el diagnóstico de demencia. Sin embargo, se debe tener presente el sesgo al excluir AM que presentaban evidencia de deterioro cognitivo. No obstante, existe certeza que tanto el nivel de escolaridad como la edad influyen en la capacidad cognitiva, lo que se ha demostrado en estudios con muestras de representatividad nacional como es la Encuesta nacional de salud y estudios internacionales $^{22}$. La depresión establecida, clasificada por la GDS alcanzó sólo 4\%; otros estudios ${ }^{14,25}$, han determinado cifras superiores. Esta baja presencia de depresión en la muestra es congruente con los altos porcentajes de percepciones de salud y memoria, en la categoría buena-muy buena, así como la escasa limitación para las actividades diarias; a pesar de la existencia de enfermedades crónicas y el consumo diario de dos a siete medicamentos $(53,1 \%)$. Además, estos datos, reflejan la importancia del "sentirse bien" más que "estar bien" 23 que declaran las personas.

Esta investigación, detectó problemas físicos y funcionales en los AM de Antofagasta. No obstante, es una visión parcial, los AM con deterioro funcional y cognitivo que no asisten a consultorios $y$ en actividades comunitarias no formaron parte de la muestra, lo que explicaría la menor frecuencia de HTA, depresión o deterioro cognitivo en relación a otros estudios nacionales. Sin embargo, este grupo evidencia problemas de salud en una muestra de AM probablemente sanos y activos, por lo tanto, es esperable un mayor deterioro funcional y cognitivo en una muestra representativa de la ciudad.

El aporte de este estudio es identificar los riesgos de deterioro asociados al proceso de envejecimiento, para posteriormente elaborar programas multidimensionales de prevención o disminución del deterioro funcional que genera dependencia, discapacidad y finalmente fragilidad ${ }^{24}$. El 56,1\% de los AM de Antofagasta tienen entre 60-69 años, el control de los riesgos de deterioro en este grupo etáreo, implica mejorar las oportunidades de alcanzar el envejecimiento activo en AM, que por proyecciones estadísticas ${ }^{25}$, pueden vivir más de 20 años.

\section{Referencias}

1. Instituto Nacional de Estadísticas. Enfoque estadístico. Julio 2007 www.INE.cl.

2. Instituto Nacional de Estadísticas. www.INE.cl.

3. Instituto Nacional de Estadísticas. Estimaciones y proyecciones de la población por sexo y edad. Regiones 1999-2010. Chile y los Adultos mayores en la sociedad del 2000. www.INE.cl

4. Corraliza J A. Vejez y Sociedad: dimensiones psicosociales. En: Gerontología social. Fernández-Ballesteros R (Editor) Madrid, España: Editorial Pirámide 2000; p 229-38.

5. Fernández-Ballesteros R. Gerontología Social. Madrid, España: Editorial Pirámide 2000; p 31-54.

6. OMS. 2004; 1-5. Disponible en: www.who.int/gb/ebwha/ 
pdf_file/EB115/B115_29-sp.pdf (Consultado febrero de 2008).

7. Montorio I, Izal M, Pérez G. Salud, conducta y vejez. En: Psicología de la vejez. Triado C, Villar F (Coordinadores). Madrid, España. Editorial Alianza 2006; p 87-109.

8. Ruipérez I. Escalas de Valoración en contextos geriátricos. En: Gerontología social. Fernández-Ballesteros R (Ed.). Madrid, España. Editorial Pirámide 2000; p 38399.

9. Tromp AM, Pluijm SM, Smit JH, Deeg DJ, Bouter LM, Lips P. Fall-risk screening test: a prospective study on predictors for falls in community-dwelling elderly. J Clinical Epidemiology 2001; 54: 837-44.

10. MIDEPLAN 2005; 1- 30. Disponible en: www.infopais. cl/ (consultado: septiembre 2009).

11. Sandis E. The aging and their families: a Cross national review En: International perspectives on human development. Comunian A; Gielen U. (Eds) Lengerich, Berlín: Pabst Science Publishers 2000; p 591-9.

12. Ministerio de Salud 2004. Encuesta de salud. Disponible en: www.ssmc.cl/documentos (consultado febrero 2009).

13. Marín P, Villalobos A, Carrasco M, Kalache A. Resultados generales del Proyecto INTRA-OMS en Chile. Rev Med Chile 2005; 133: 331-7.

14. Díaz V, Díaz I, Rojas G, Novogrodsky D. Evaluación geriátrica en la atención primaria. Rev Med Chile 2003; 131: 895-1.

15. Alonso P, Sanso F, Díaz Camel A, Carrasco M, Oliva T. Envejecimiento poblacional y fragilidad en el adulto mayor. Rev Cub Salud Pública 2007; 33. www.Scielo.org (consultado febrero 2009).

16. PAHO.org. 2001. Encuesta SABE. Disponible en: www. paho.org/spanish (consultado febrero 2009).

17. Séculi E, Brugulat P, March J, Medina A, Martínez V,
Tresserras R. Las caídas en las personas mayores de 65 años: conocer para actuar. Aten Primaria 2004; 34: 18690.

18. Corrales D, Palomo L, Magariño M, Alonso G, Torrico $\mathrm{P}$, Barroso A, et al. Capacidad funcional y problemas socioasistenciales de los ancianos del área de salud de Cáceres. Aten Primaria 2004; 33: 426-33.

19. Azpiazu M, Cruz A, Villagrasa J, Abanades J, García N, Álvarez C. Calidad de vida en mayores de 65 años no institucionalizados de dos áreas sanitarias de Madrid. Aten Primaria 2003; 31: 285-92.

20. Quiroga P, Albala C, Klaasen G. Validación de un test de tamizaje para el diagnóstico de demencia asociada a la edad. Rev Med Chile 2004; 132: 467-75.

21. Manual Diagnóstico y Estadístico de los Trastornos Mentales. (DSM IV- TR). Edición en español. 4 ed. Barcelona: Elservier Masson; 2007.

22. Gázquez JJ, Rubio R, Pérez MC, Lucas F. Análisis de los factores predictores de la dependencia funcional en personas mayores. International Journal of Psychology and Psychological Therapy 2008; 8117-126. Disponible en: http://redalyc.uaemex.mx/redalyc/src/inicio/ArtPdfRed.jsp?iCve $=56080110$ (Consultado el 20 de agosto de 2009).

23. Gayoso P. La calidad de vida relacionada con la salud. Importancia en atención primaria. Aten Primaria 2003; 31: 293-4.

24. Rodríguez L, Castro M. Envejecimiento y fragilidad. En: Síndromes y cuidados en el paciente geriátrico. Guillén F, Molino J y Petidier R. Elsevier Masson. Barcelona, España. 2008; p 21-31.

25. INE. Documento de trabajo. Perfil Epidemiológico del adulto mayor en Chile. 2006 www.supersalud.cl/documentacion/569/articles-4020_recurso_1.pdf. 